\title{
SIMULATIONS OF HST OBSERVATIONS OF GLOBULAR CLUSTERS
}

\author{
John N. Bahcall and Donald P. Schneider
}

Institute for Advanced Study

\section{INTRODUCTORY REMARKS}

The high angular resolution of the Hubble Space Telescope will provide opportunities for many fundamental observations of globular clusters, most of which have been extensively discussed in the literature. We have therefore chosen to devote our time (and pages) to a presentation of what HST observations may reveal about some aspects of galactic globular clusters. To avoid infringing upon programs that others may propose, we have limited ourselves to simulations of observations that are part of our Guaranteed Time Observations. [The complete catalog of GTO observations has published by the Space Telescope Science Institute and is available upon request.]

The science programs described here are: 1) studies of the cores of nearby galactic globular clusters (exemplified in what follows by pictures of M13); and 2) stellar population studies of a nearby relatively unobscured globular cluster (exemplified in what follows by pictures of NGC 6397). The purposes of the first program include the determination of the stellar density distributions and luminosity functions in the innermost regions of the globular clusters. The second project is a joint investigation with the WF/PC instrument development team and is aimed at detecting white dwarfs and the faint red end of the cluster luminosity function.

\section{WHAT IS INCLUDED IN A SIMULATION?}

The simulations we present were created using the image processing code developed by one of us (DPS) and refer to the Wide Field/Planetary Camera (Westphal et al. 1982) operating in the Wide Field mode. The Wide Field Camera (WFC) has a field of $160^{\prime \prime}$ on a side and an image scale of $0.1^{\prime \prime}$ per pixel. Because of hardware limitations, we display only a 500 pixel by 500 pixel subregion of a WFC frame. This is not an important constraint for the purposes of this paper, since our goal is to present a qualitative demonstration of some of the ways that HST observations can supplement ground-based studies.

The recently updated instrument and telescope parameters (Westphal et al. 1986) were used to construct the HST pictures. The instrumental parameters required for the simulations include the point spread function, the quantum efficiency, the filters, the sky background, and the instrumental noise. For the clusters, we need the stellar luminosity function (taken from Bahcall 1985), a distribution of stellar types with absolute magnitude (adapted from Bahcall and Soneira 1984), the central surface brightness, the core radius. the distance, and the reddening. The 
observational parameters were taken from Peterson and King (1979) and Harris and Racine (1979). We have purposely chosen not to use the latest and most detailed parameterizations from the ground based data, in order that our simulations be properly regarded as only illustrative. Since the dynamic range of the detectors in the WFC far exceeds that of a photograph, the visual appearance of the pictures depends strongly upon what range of intensities (the "stretch") of the data are selected to be displayed. We have made pictures with a variety of stretches and present here the images that we believe are the most representative and revealing.

\section{THE PICTURES}

The only real data we show is a picture of M13 (distance $6.1 \mathrm{kpc}$ ) which is displayed in Figure 1 (and again in zoom-format in Figure 2); this image was obtained with a 10 second exposure using the "4-Shooter" camera (Gunn et al. 1984) and the $\mathrm{r}$ filter on the Palomar 5 meter telescope. The image displays the inner regions of this bright globular cluster; the figure is $250^{\prime \prime}$ on a side. The exposure was acquired in excellent seeing; the full-width at half-maximum of the stellar images is $0.8^{\prime \prime}$. The limiting visual magnitude corresponds to $m_{V} \sim 22.5^{m}$.

In order to have a ground-based image that is on the same scale as the HST simulations we will show later, we display in Figure 2 the inner $50^{\prime \prime} \times 50^{\prime \prime}$ of Figure 1. Notice that the images appear to be almost merging blobs, even with excellent seeing conditions.

The first "HST" image is shown in Figure 3. This simulation represents a 10 second exposure of the core of M13 taken with the (wide visual) F555 W filter. There are approximately $10^{3}$ stars visible down to the limiting magnitude of $m_{V} \sim 22.5^{\mathbf{m}}$.

How would the field of Figure 3 appear if it had been observed in $0.8^{\prime \prime}$ seeing with the same filter? Figure 4 shows the simulated ground-based image. Note that enormously fewer stars are visible and that many of the images that are clearly separated in Figure 3 have merged to form amorphous blobs in Figure 4. Many of the bright "stars" in Figure 4 are really small stellar groups (cf. Figure 3), which suggests caution is required in interpreting ground-based observations of dense cluster cores. The simulated ground-based picture shown in Figure 4 is similar to what has been obtained under good conditions by observers who have studied the cores of globular clusters.

HST observations of the inner regions of globular clusters will make important contributions to the observational understanding of core collapse and mass segregation.

Figures 5 and 6 show HST images of NGC 6397 (distance $2.1 \mathrm{kpc}$ ). The ultraviolet exposure is shown in Figure 5 and represents a 45 minute (full-orbit) image taken with the F336M filter (effective wavelength about $3360 \AA$ ). The limiting visual magnitude is about $m_{V} \sim 27^{m}$. The I-band picture is shown in Figure 6 and represents a 7.5 minute exposure with F785LP; it reaches to about $m_{I} \sim$ $26^{m}$. Saturation of the CCD charge storage capacity by bright stars creates the ugly streaks that are so prominent in Figure 6 and which, unfortunately, will be a common occurence of HST images of globular clusters. It will not be easy to perform accurate photometry with such data, especially when one considers that the images are severely undersampled.

We hope to observe $\sim 10^{2}$ white dwarfs on the full WFC image. A rather different estimate has been given by Renzini (this conference), who based his results on theoretical stellar evolution calculations. With the many uncertainties (theoretical 
and observational, including cooling; see Bahcall 1985) that enter such predictions, an uncertainty in the estimated number of white dwarfs of order a factor of 5 or even 10 is not surprising. The first HST observations of white dwarfs may provide important constraints on calculations of the advanced stages of stellar evolution.

We also expect to observe $\sim 10^{4}$ faint red dwarfs (on the full WFC image) in an effort to determine the low-mass end of the cluster luminosity function, and with luck, obtain some insight into the initial mass function of globular clusters.

The images shown in Figures 1-6 give what we believe is a representative impression of the potentialities of the HST for studies of the cores of galactic globular clusters and for measuring the faint ends of the cluster luminosity functions.

This work was supported in part by NASA grants NAS8-32902 and NAS5-29225.

\section{REFERENCES}

Bahcal1, N. N. and Soneira, R. M. 1984 Astrophys. J. 55, 67.

Bahcall, J. N. and Soneira, R. M. 1985 in IAU Symposium No.

113. Dynamics of Star Clusters, J. Goodman and P. Hut, eds., Reidel, Dordrecht, p. 481.

Gunn, J. E. 1984 Bul1. A. A. S. 16, 477.

Harris, W. E. and Racine, R. 1979 Ann. Rev. 17, 241.

Peterson, C. J. and King. I. R. 1975 Astron. J. 80, 427.

Westphal, J. A. and WF/PC IDT Team 1982 in The Space Telescope

Observatory, IAU 18th General Assembly, D. B. Hall, ed., NASA CP 2244, Washington, p. 28.

Westpha1, J. A. and WF/PC IDT Team 1986, private communication. 


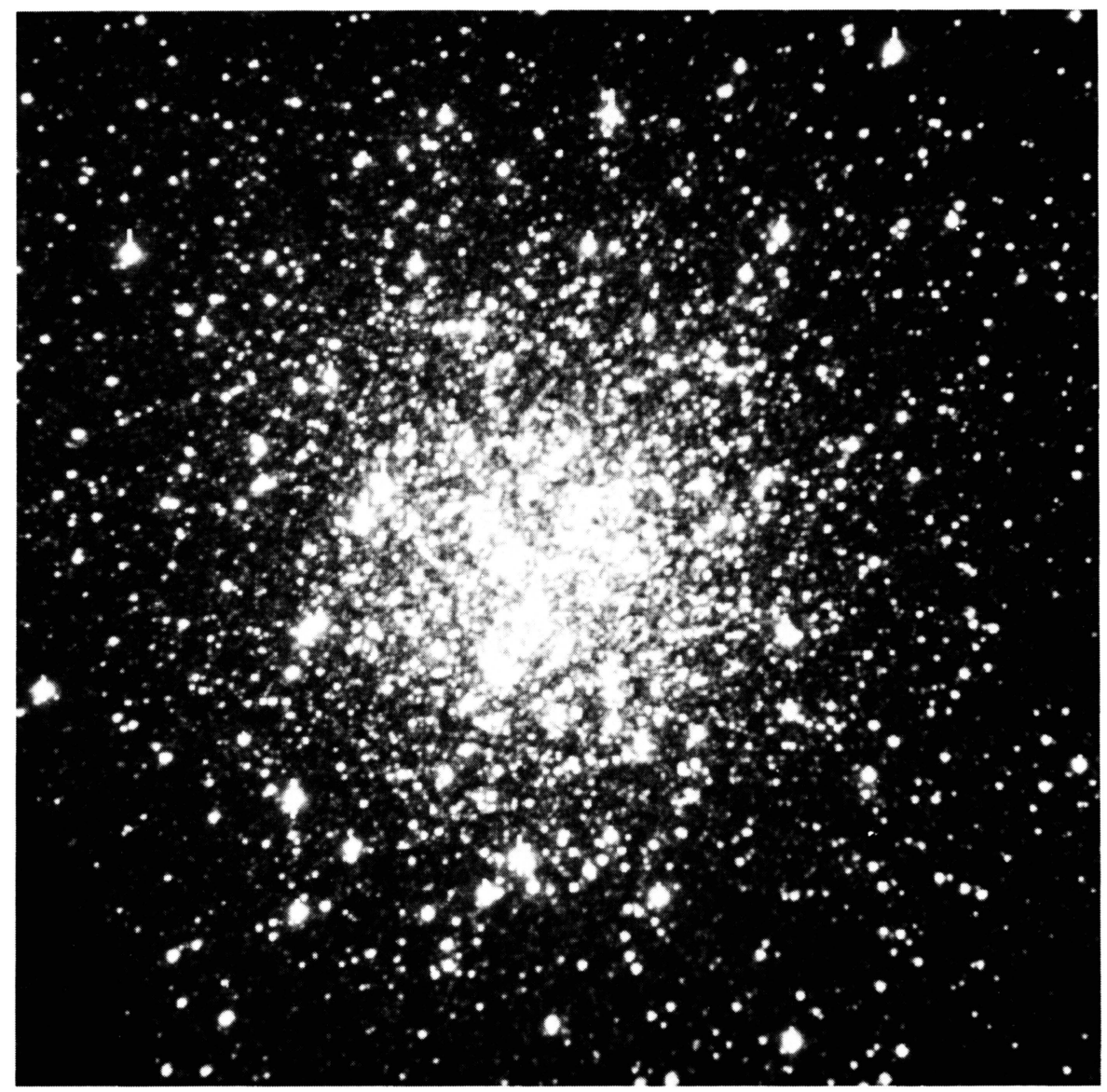

Fig. 1. A Palomar 5 meter Image of the Inner Region of M13. The exposure was obtained with excellent seeing using an $\mathrm{r}$ filter. 


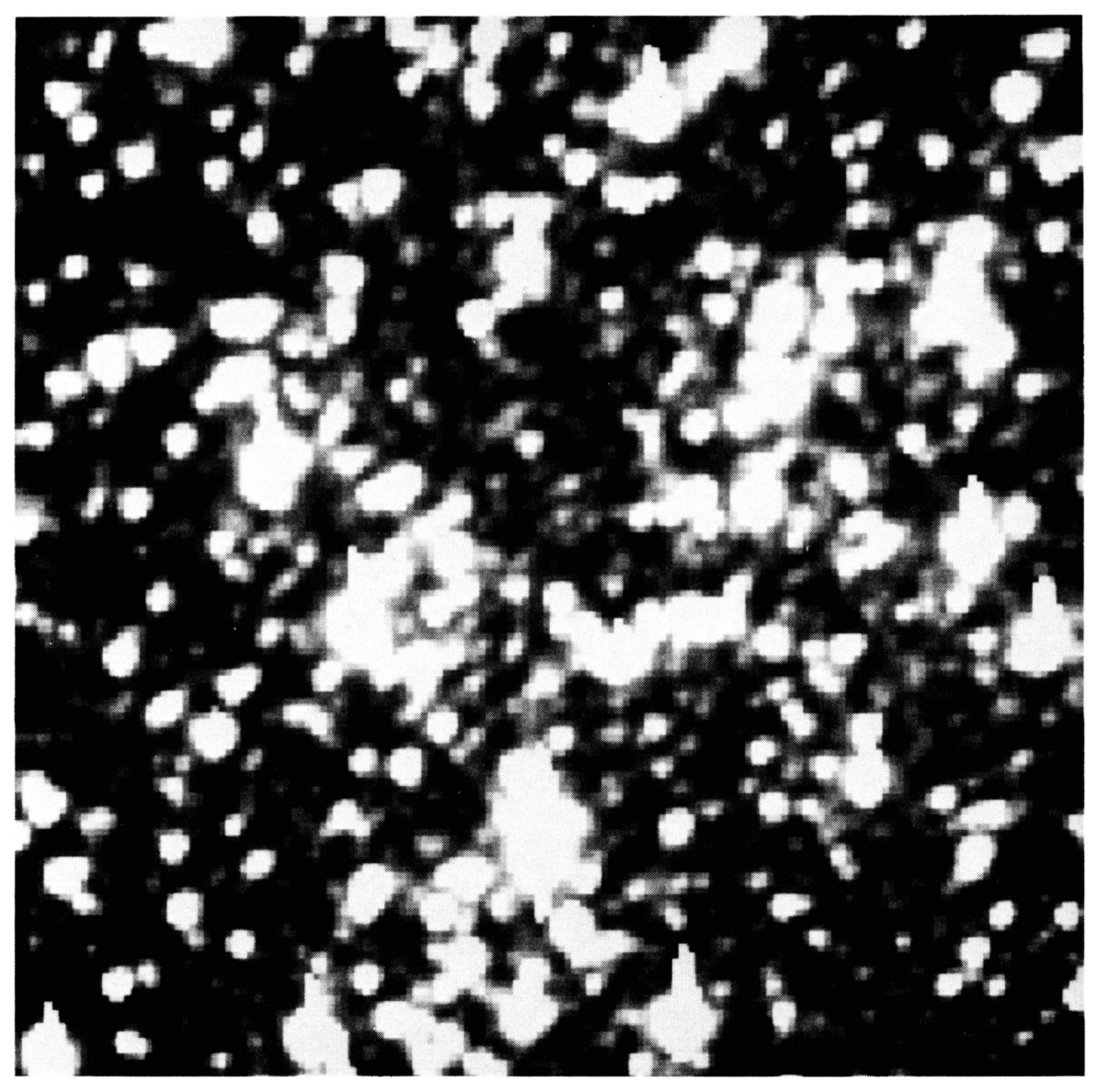

Fig. 2. The Innermost $50^{\prime \prime} \times 50^{\prime \prime}$ of Figure 1 . 


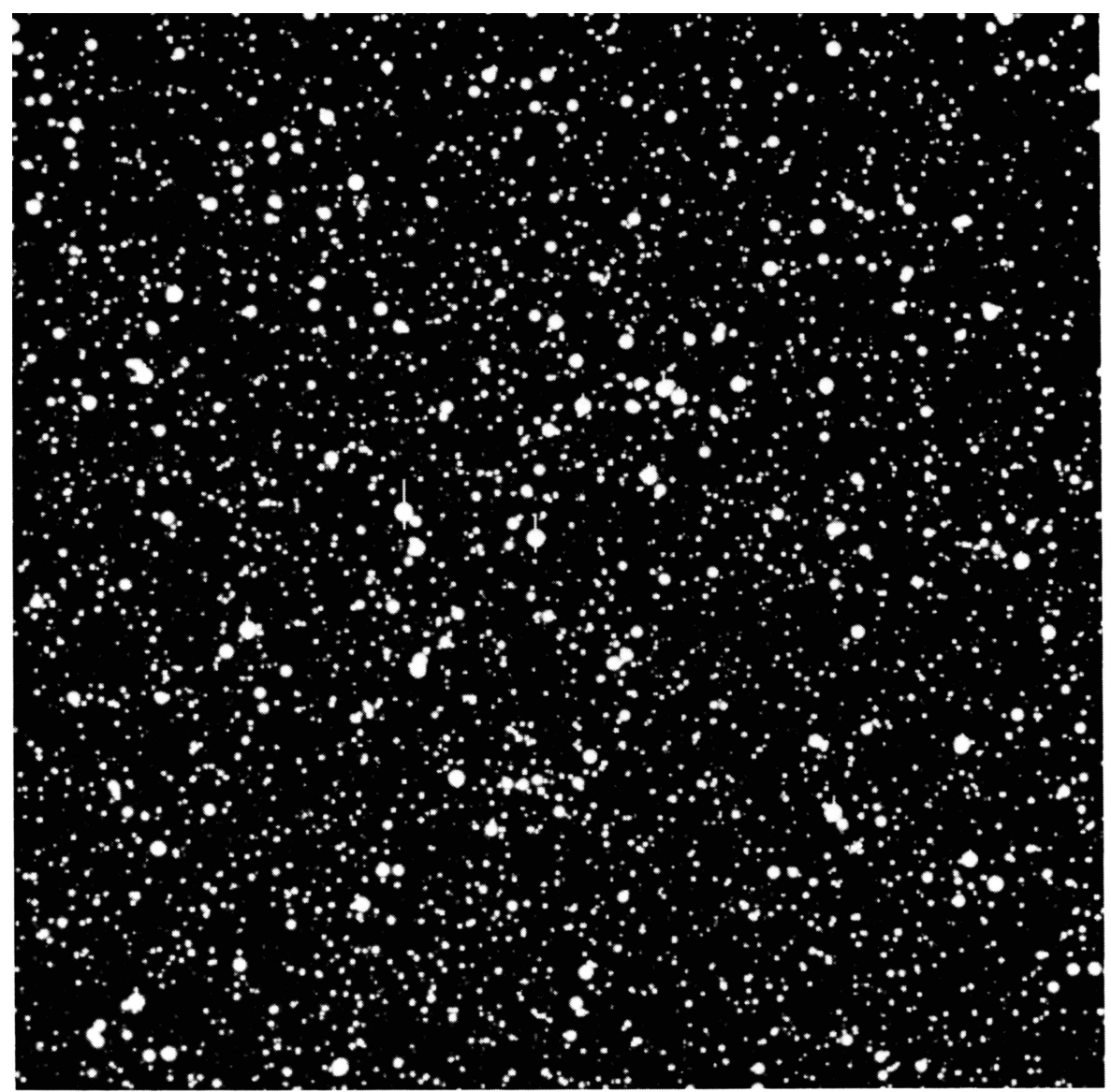

Fig. 3. A Simulated HST Picture of the Core of M13 with a Visual Filter. The field is $50^{\prime \prime} \times 50^{\prime \prime}$. 


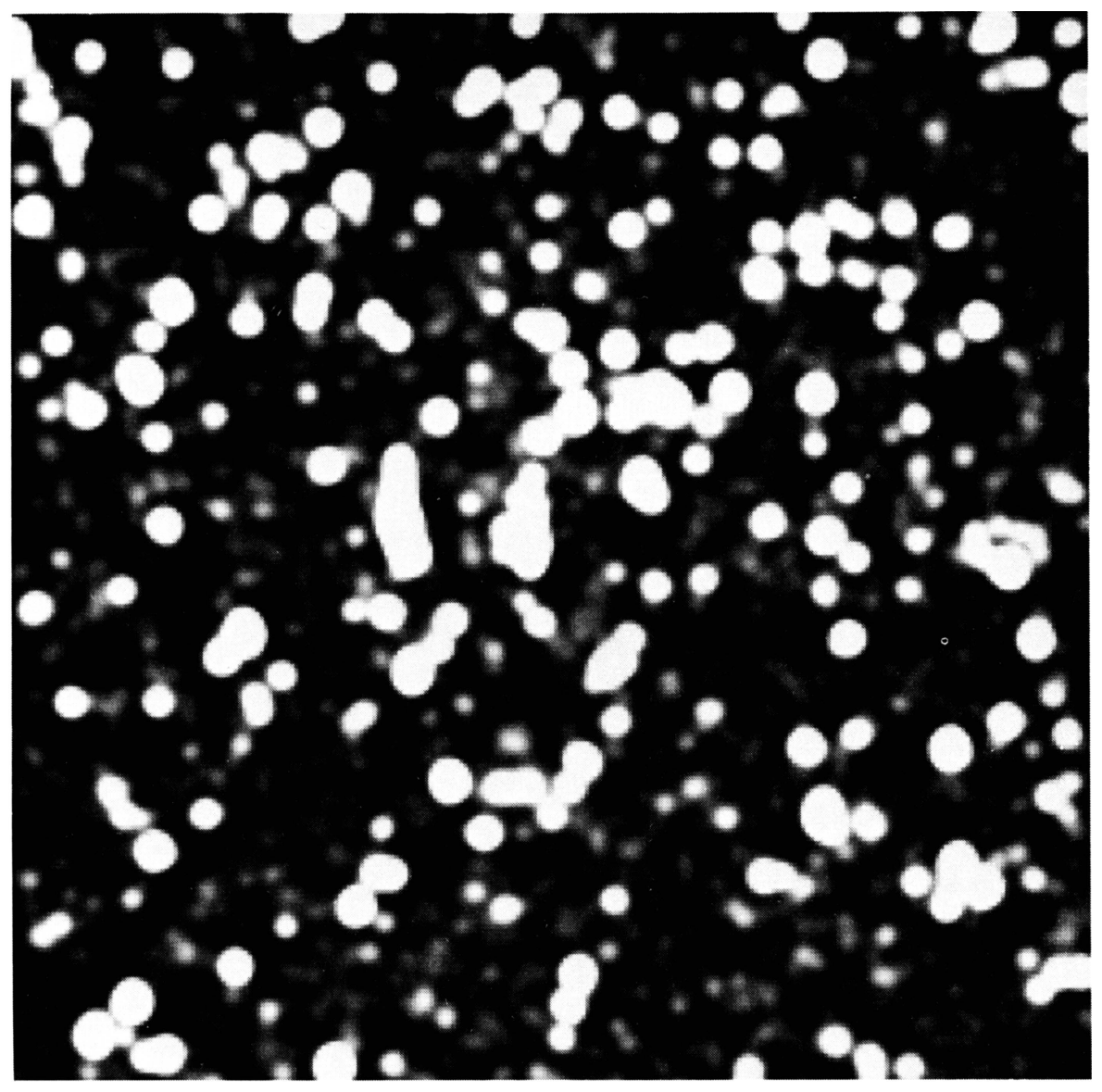

Fig. 4. The Field of Figure 3 "Observed" with 0.8 " Resolution. 


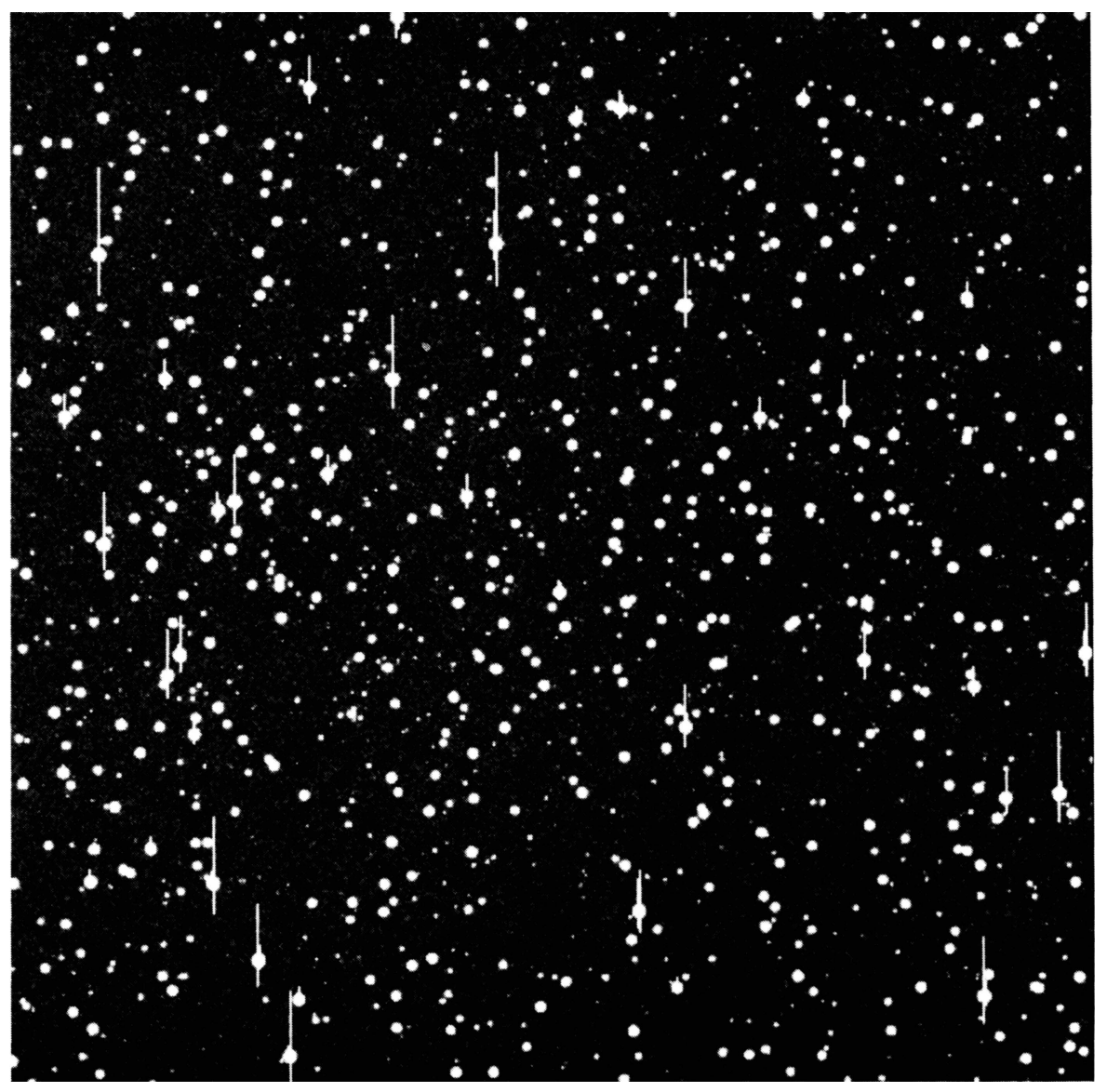

Fig. 5. A Simulated Ultraviolet HST Exposure of the Core of NGC 6397. 


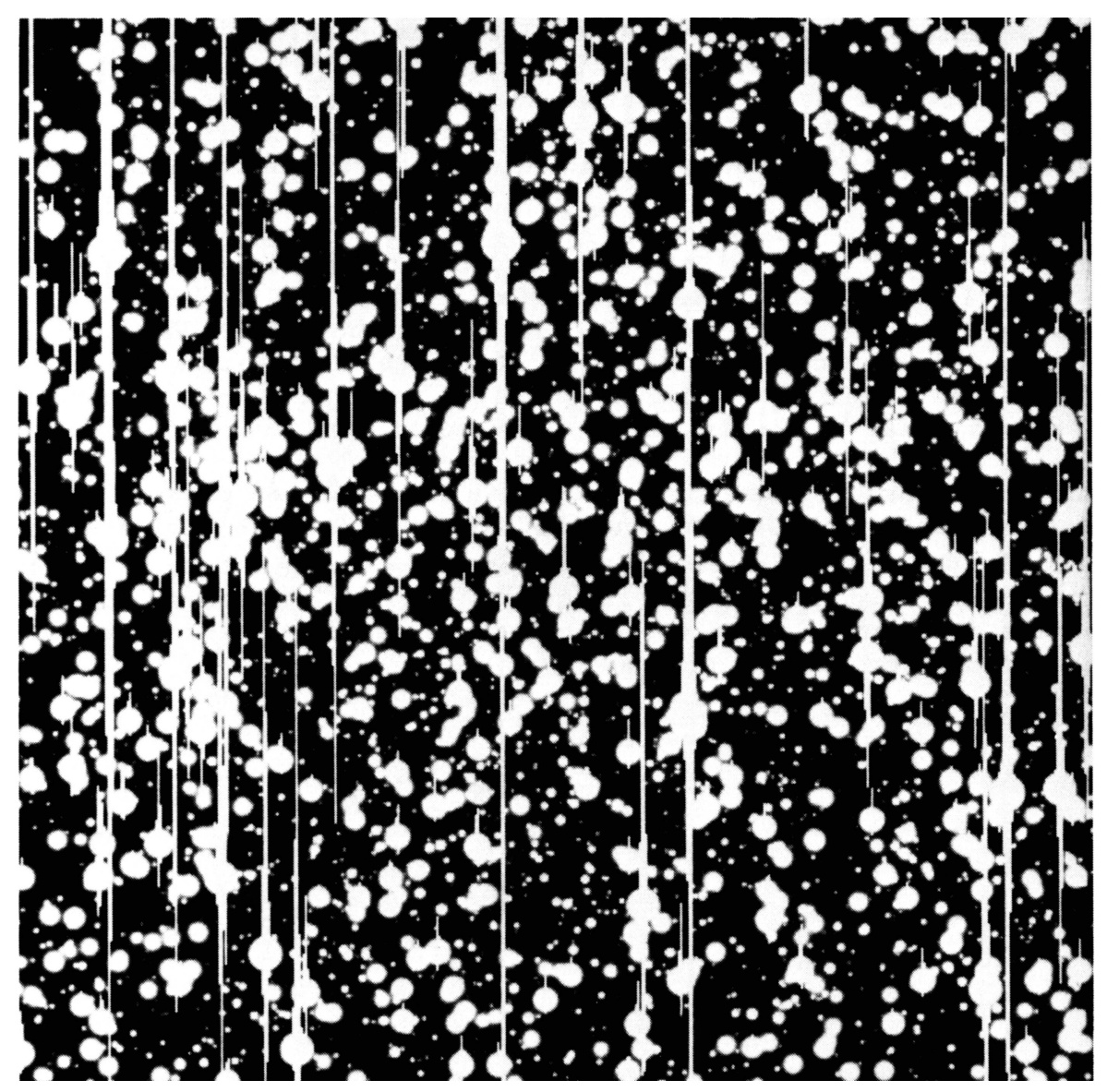

Fig. 6. A Simulated I-band HST Exposure of the Core of NGC 6397. 


\section{DISCUSSION}

CHRISTIAN: While it is appreciated that we should carefully consider the expected performance of the HST, and that comparison of existing ground based data to HST data will be interesting if not problematic. I think the capabilities of ground based facilities have been underestimated in this talk. At CFHT we have obtained images with FWHM of $0.25 \mathrm{arc} \mathrm{sec}$. If we had available the HST budget for 2 months we could commission such facilities such as adaptive optics, tip-tilt mirrors and fast shutters: then we would be able to achieve very high resolution (spatial) routinely in the next few years before HST is launched.

COHEN: The 200" inch mirror is only good to $0.6 "$, based on Hartmann tests. I agree with everything Carol Christian said. The base atmosphere above a high site has a PSF with about 0.3" FWHM. All new ground-based telescopes will be built to take advantage of the best ground-based seeing. Image profiles on existing ground-based telescopes could be significantly improved if funds were available.

KING: May I briefly describe the FOC Guaranteed Time program? In five globular clusters ( $\omega$ Cen, 47 Tuc, NGC 6397, 6624 and 6752) we will take one orbit $B$ and $V$ exposures at the center, $1 r_{c}$ and $3 r_{c}$. The outer parts should be done from the ground. We will also look at the central profiles of about a dozen collapsed-core clusters. In M 31 we have 14 globular clusters on the program. 